\title{
Intervention to reduce procrastination in first-year students: Preliminary results from a Norwegian study
}

psykologisk.no/sp/2016/06/e10/

\section{Our study indicates that a brief intervention program for students may help reduce academic procrastination, write Kent Nordby and colleagues.}

BY: Kent Nordby, Catharina Elisabeth Arfwedson Wang, Tove Irene Dahl and Frode Svartdal

Procrastination is one of the most challenging problems facing students today. Procrastination refers to the voluntary delay of intended tasks despite expecting to be worse off because of the delay (Steel, 2007). Students are especially prone to dilatory behavior, with prevalence estimates ranging from $30 \%$ to $60 \%$ (Rabin, Fogel, \& NutterUpham, 2011) or even higher (Steel, 2007). In contrast, the prevalence in the normal population is $20 \%$ (Steel, 2007). In students, procrastination manifests itself as a delay in academic tasks such as writing term papers, studying for exams, and doing other necessary coursework. More than half of all students express a need for help in handling their procrastination (Gallagher, Golin, \& Kelleher, 1992). Procrastination affects academic performance negatively (Steel, 2007) and is also related to increased stress (Sirois, 2014), depression and anxiety (Flett, Blankstein, \& Martin, 1995; Stöber \& Joormann, 2001), worry (Antony, Purdon, Huta, \& Swinson, 1998; Ferrari, Johnson, \& McCown, 1995), and shame and guilt (Fee \& Tangney, 2000; Pychyl, Lee, Thibodeau, \& Blunt, 2000). Procrastinators report lower levels of self-compassion, suggesting that self-kindness and self-acceptance may be difficult for those who needlessly delay (Sirois, 2014). In sum, procrastination may be a serious problem for students, and its seriousness escalates as the procrastination increases.

Personality traits such as high impulsivity, low self-control, and low conscientiousness predict procrastination (Gustavson, Miyake, Hewitt, \& Friedman, 2014; Steel 2007). Procrastination has been described as a prototypical example of failure in selfregulation (Steel, 2007) and is also often regarded as a strategy to escape negative emotions such as stress and anxiety-i.e., "giving in to feel good" (Tice \& Bratslavsky, 2000). The tendency to procrastinate is especially likely when dealing with tasks that have long or unclear deadlines (Ariely \& Wertenbroch, 2002; Schraw, Wadkins, \& Olafson, 2007) and when tasks are boring or aversive (Steel, 2007). Pychyl and colleagues (2000) found that boring or aversive tasks increased the likelihood of engaging in distracting behaviors such as watching television, playing on the computer, and chatting with friends, indicating that procrastination involves both the delay of intended tasks and a preference for alternative short-term pleasurable activities. It is 
not surprising, therefore, that high procrastination is associated with a low degree of self-determined motivation (Lee, 2005; Steel, 2007).

A large number of self-help books, Internet resources, and theoretical papers describe possible methods to reduce procrastination, yet the number of studies demonstrating effective interventions is relatively low. One recommended intervention strategy is teaching better time management, i.e., skills and behaviors relating to task and time awareness, planning, goal setting, prioritizing, scheduling, organizing, and establishing new and improved time habits (Hellsten \& Rogers, 2009; Karas \& Spada, 2009; Schmitz \& Wiese, 2006). For example, Häfner, Oberst, and Stock (2014) administered a four-hour time management program that taught undergraduates how to better organize their time. It included modules focusing on implementation intentions, prioritizing, monitoring, and structuring of their workday. After one month, participants demonstrated a significantly better distribution of workload across the term compared to control students.

Other studies have indicated positive effects of setting deadlines (Ariely \& Wertenbroch, 2002; Lamwers \& Jazwinski, 1989; Roberts, Fulton, \& Semb, 1988), imposing working time restrictions (Höcker, Engberding, Haferkamp, \& Rist, 2012), implementing enforced daily writing sessions (Boice, 1989), and controlling stimuli such as removing distractions from the work area (Ziesat, Rosenthal, \& White, 1978). Promising results have also been found from a short mindfulness group-training intervention (Scent \& Boes, 2014).

Because procrastination has been connected to dysfunctional beliefs such as unrealistic expectations, low self-efficacy, and negative thoughts (Pychyl \& Flett, 2012), the use of cognitive behavioral therapy (CBT) has been suggested as a remedy. In $\mathrm{CBT}$, procrastinators are taught to dispute their irrational thoughts and beliefs, develop goals, and develop a mindset that enables them to persist better in doing aversive tasks (Neenan, 2008). Rozental et al. (2015) developed an online 10-week treatment program for participants with clinical levels of procrastination. Two groups receiving guided or non-guided self-help interventions demonstrated a significant reduction in procrastination at post-treatment and follow-up compared to a control group. Other intervention studies have found that more traditional in-vivo group CBT also reduces procrastination (Ozer, Demir, \& Ferrari, 2013).

Overall, intervention studies such as those discussed indicate that procrastination can be ameliorated. In addition to reducing procrastination, other beneficial effects of interventions include a more positive attitude toward studying (e.g., Ziesat, Rosenthal, \& White, 1978), better grades (Tuckman, 1998; Wesp, 1986), and reduced stress (Wäschle et al., 2014).

\section{The present study}

The purpose of the present study was to assess the usefulness of a brief intervention against procrastination for Norwegian students. The need for such an intervention, 
particularly among beginner students, is obvious. At least one-third of college and university students demonstrate procrastination levels that significantly handicap them in their work, both in the short-term and the long-term perspectives (Botnmark, Kvalnes, \& Svartdal, 2014). Ideally, interventions should address such groups specifically (Rozental et al., 2015), but a more practical approach is to present intervention or prevention efforts for larger groups of students as part of their normal curriculum (e.g., Tuckman \& Kennedy, 2011). Although such an approach limits the possibility of randomizing participants to conditions, it still can provide valuable information about factors associated with positive change. Such general interventions would also be feasible, especially for introductory psychology students. That is because the topics addressed are easily included in their regular curriculum. Hence, the present intervention was implemented as a part of psychology students' regular course of study. It consisted of lectures and seminar sessions given over a four-week period as well as reading material related to procrastination and techniques to overcome the issue.

The course included a combination of elements previously found to be effective in reducing academic procrastination: (1) Information about the procrastination habit, and its causes and consequences. Special emphasis was put on the importance of selfawareness (i.e., identifying behaviors, irrational thoughts, and emotions related to procrastination), because increased attention has been found to strengthen selfregulation (Baumeister \& Heatherton, 1996); (2) Information related to time management skills and planning, with a focus on task and time awareness, planning, goal setting, and establishing improved time use habits; and (3) Cognitive behavioral therapy techniques (Rozental et al., 2015), including the management of thoughts and feelings (e.g., negative or irrational thoughts, failure, guilt, shame, and stress) related to dilatory behavior.

To assess changes related to the intervention, scales measuring procrastination and time management skills were administered in a simple pre/post design. Overall, we expected the intervention to be associated with a reduction in procrastination scores. We also anticipated that students with higher levels of procrastination would benefit more from the course because students with high procrastination levels are often motivated to change (Onwuegbuzie, 2004). We included two procrastination scales, one rather abstract and trait-oriented, the other more concrete and behavior-oriented. Both scales should demonstrate changes associated with the intervention, but the behavior-oriented scale was expected to be more sensitive in capturing short-term changes in procrastination. Because time management skills are closely related to procrastination, we expected changes in such skills to mirror eventual changes in procrastination scores. A questionnaire measuring positive motivation to study was also administered. Because procrastination is negatively related to positive motivation (Lee, 2005), students with low motivation were expected to demonstrate higher procrastination scores at pre-test. However, because the role of motivation in bringing about positive change in procrastination is uncertain, this issue was further explored in 
the analyses.

\section{Method}

\section{Participants}

From a pool of approximately 300 students who were enlisted in an introductory psychology course and enrolled in the autumn term at a university in Norway, 219 students (164 females) participated. A majority were first-year students, mean age $=$ 21.7 years $(S D=4.33)$. All participated in exchange for joining a lottery with a chance to win a digital tablet or mobile phone.

\section{Instruments}

Irrational Procrastination Scale (IPS). The IPS (Steel, 2010) is a self-reported scale measuring habitual delay in behavioral implementation. The scale contains items from three established procrastination scales and is assumed to measure a unidimensional construct, "irrational delay," with "irrational" referring to the delay as disadvantageous to the person and further that the person also realizes this fact (Steel, 2010). IPS consists of nine items (e.g., "I put things off so long that my well-being or efficiency unnecessarily suffers"), three of which are inconsistent with procrastination and thus are reversed before analysis. All items are rated on a five-point Likert-scale (1-5), with higher scores indicating more problems with procrastination. The Norwegian version was translated and validated by Svartdal (2015), with good internal consistency (Cronbach's $\alpha=.85-.91$ ). In the present study, Cronbach's $\alpha$ at pre-test was .92.

Self-Regulation Scale (SRS). The Self-Regulation Scale (Job, Walton, Bernecker, \& Dweck, 2015) is a self-reported scale that was developed to measure everyday selfregulation failure during the previous week (27 questions). Six of these items were selected for the present study, all of which relate to specific examples of procrastination during the previous week (e.g., Items 1 and 2: "During the last week: How often did you delay doing something for your studies, even when it was important?"; "How often did you postpone starting studying, even if it would have been important to start?"). The questions are answered on an ordinal seven-point scale, with 1 being "Never" and 7 being "Twice or more per day." Cronbach's a for the procrastination part of the SRS is $\alpha=.78-.87$, and the items seem to measure a unidimensional construct (Haug, 2016; Job et al., 2015). In the present study, Cronbach's a was .81 at pre-test. Previous studies have indicated that this procrastination scale correlates highly with IPS (Haug, 2016), $r=.53-.68$. Of importance here is that IPS measures procrastination as a relatively stable trait, whereas the SRS addresses specific examples of procrastination behavior occurring in the past week. For this reason, the latter scale should be superior in measuring changes in procrastination behavior.

Time Management Questionnaire (TMQ). This questionnaire was developed by Britton and Tesser (1991) to evaluate students' use of time-management techniques. It is 
composed of 35 items covering three aspects of time management (short-range planning, time attitudes, and long-range planning) and is answered on a five-point Likert-scale (1-5). For this study, we selected the seven items from the short-range planning subscale (e.g., "Do you make a list of the things you have to do each day?"). Britton and Tesser (1991) identified these items to constitute one of three factors in the TMQ. Cronbach's a for these items at pre-test was .87.

Motivational questionnaire. We created four questions related to positive emotions toward course and schoolwork (e.g., "I enjoy working on the subject" and "I always do my best when working on a subject"). The purpose of these questions was to address the degree of positive motivation toward studying, the coursework, and the subject in general. Cronbach's $\alpha$ for these items at pre-test was .80 .

\section{Intervention}

The first lecture introduced the procrastination problem and how it manifests itself. Emphasis was put on the fact that procrastination is a negative habit that can be changed. In the next two lectures, students were guided in techniques of task and time management, the importance of using calendars, and suggestions on how to structure and monitor their daily activities. Additionally, students were encouraged to start using to-do lists and to set appropriate deadlines for themselves. They were also taught how to better evaluate the duration of academic tasks. Topics related to change (e.g., fixed vs. growth mindset) were discussed as well. In the final lecture, students were introduced to cognitive behavioral therapy (CBT), explaining how thoughts, feelings, and behavior interplay in procrastination. After the last week of lectures, students had the option to participate in a two-hour seminar. Here students could practice using techniques from the lectures as well as prepare for exam questions related to the contents of the intervention.

\section{Procedure}

The intervention had a duration of four consecutive weeks in the middle of the autumn term, with a total of four lectures and two seminars. Students answered a web-based questionnaire (www.qualtrics.com) before the first lecture (pre) and then again one week after the final lecture (post). Participation was voluntary and responses were given anonymously. Before getting access to the questionnaires, the students received information regarding the study's purpose. By starting the questionnaires, they gave active consent to be included in the study. The project of which the current study is part received ethical approval from the Regional Ethical Board in Troms $\varnothing$, Norway (REK nord 2014/2313).

\section{Results and discussion}

Procrastination. Of primary interest in this context was whether procrastination scores changed from pre-test to post-test. The IPS data were analyzed with repeated 
measures ANOVA, with time (pre vs. post) as the repeated measures factor. The ANOVA indicated a significant overall effect of time, $F(1,108)=5.48, p=.021$. A more specific test of change would be to compare pre/post levels in low (IPS score <2.5), medium (IPS scores of 2.5-3.5), and high (IPS scores > 3.5) procrastinators. The expectation here was that students high in procrastination (IPS score $>3.5$ ) would demonstrate positive change. This predicted change would manifest itself as a procrastination level * pre/post interaction effect. This expectation was supported by the ANOVA since the interaction between procrastination level and pre/post change was significant, $F(2,106)=5.31, p=.006$. Participants with IPS scores $>3.5(n=30)$ demonstrated a reduction in procrastination from 3.97 to 3.62 . Contrast analysis confirmed that this change was significant, $F(1,106)=15.31, p=.00016, n^{2}=.25$, a medium to large effect size. This change is shown in Figure 1, leftmost panel. A corresponding analysis of the SRS procrastination scores indicated similar reductions, with an significant overall pre vs. post effect, $F(1,112)=7.52, p=.007$. The planned contrast for the high procrastination group was also significant, $F(1,108)=11.76, p=$ $.0009, n^{2}=.18$. Overall, these results demonstrate that both procrastination measures indicated significantly positive pre/post changes, with a pronounced reduction in both procrastination measures for participants with high initial procrastination scores. Both procrastination measures seemed to be equally sensitive to pre vs. post changes. Because the pre/post change in procrastination scores could be explained in terms of selection effects, we compared the procrastination levels between students completing the post phase of the study vs. students who did not complete that phase, and no difference at pre-test appeared, $F(1,189)=.100, p=.75$. 


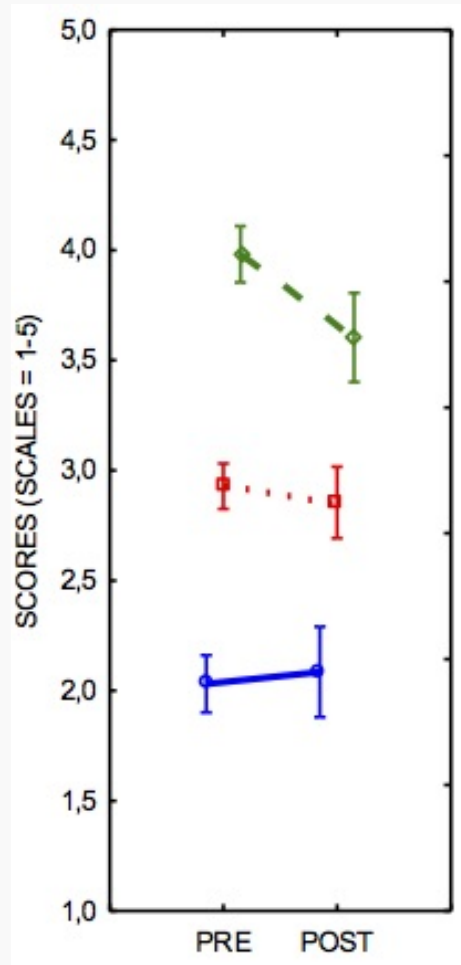

PROCRASTINATION

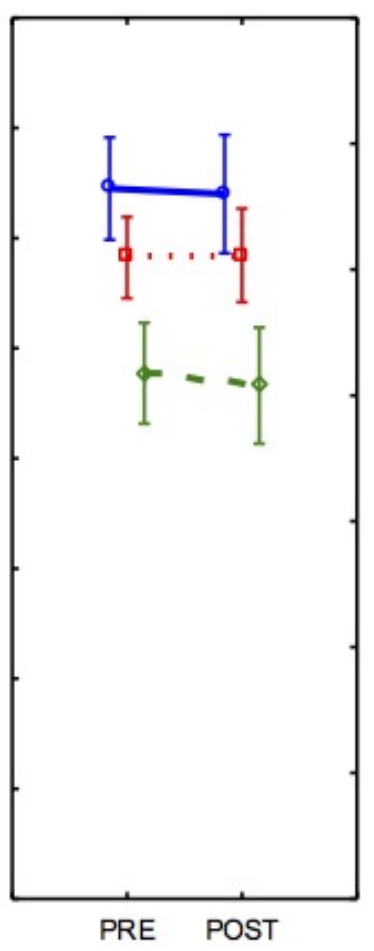

MOTIVATION

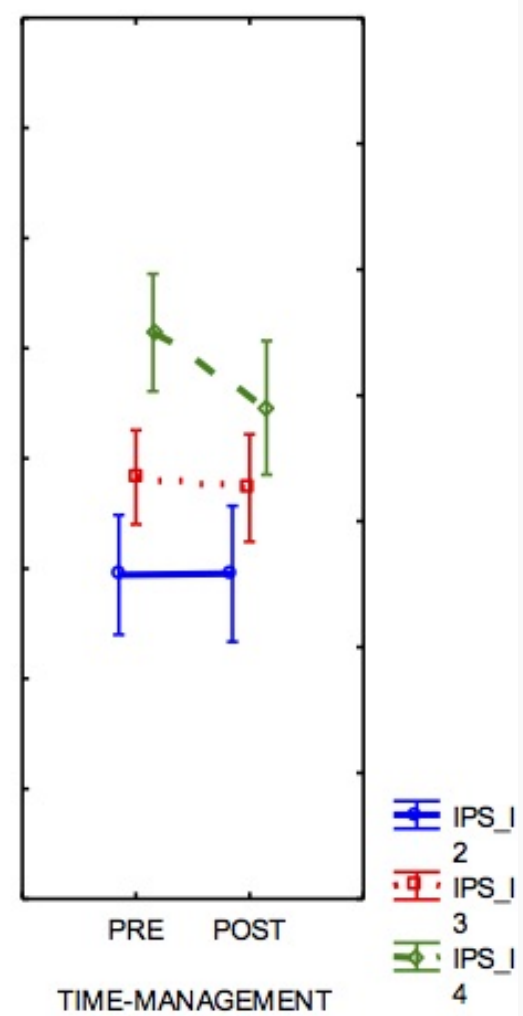

TIME-MANAGEMENT

FIGURE 1: Pre vs. post levels on the IPS (left panel), motivation questionnaire (middle panel), and TMQ (rightmost panel) for students at different procrastination levels at pre-test.

Time management skills. A corresponding analysis of changes in TMQ scores indicated a non-significant overall pre/post effect, $F(1,108)=3.24, p=.075$, but a significant change in students with initial high procrastination levels occurred, $F(1$, $106)=8.04, p=.005$. These results are displayed in Figure 1, rightmost panel.

Motivation. Because none of the intervention components addressed motivationenhancing topics specifically, no change in motivation scores between pre and post probes was expected, and none occurred, $F(1,108)=0.11, p=.74$. Even the group highest in procrastination, while demonstrating positive changes in procrastination and time management skills from pre-test to post-test, did not demonstrate any change in motivational scores. Students with low motivation were expected to procrastinate more than students with high motivation. This expectation was confirmed as the correlation between motivation and IPS scores, both at pre-test, was high, $r=-.50$. Hence, students low in procrastination (IPS score <2.5) demonstrated the highest mean motivation score (mean $=4.21$ ), whereas those with medium and high procrastination scores had means of 3.91 and 3.36 respectively.

Relation between pre/post differences in time management and procrastination. To assess possible factors associated with the observed reduction in procrastination, reduction as measured by the IPS pre/post measures were entered into a stepwise multiple regression analysis, with change scores in motivation and TMQ as predictors. Sex and age were also entered in the analysis. TMQ and motivation change scores 
were first entered, indicating an $\mathrm{R}^{2}=.14$, with $\mathrm{TMQ}$ as the best predictor, $t=3.59, p=$ .0005 . Adding sex and age into the model did not change this conclusion. Finally, analyzing the correlations between change scores of TMQ, IPS, and SRS for the three groups of procrastinators (low, medium, and high), a difference in outcome was indicated: Whereas high procrastinators demonstrated a strong correlation between TMQ and IPS changes $(r=.52)$, low procrastinators did not $(r=.09)$. The reverse trend was observed for SRS: Low and medium procrastinators demonstrated a stronger correlation between TMQ and SRS ( $r=.41$ and .35), whereas high procrastinators demonstrated a lower correlation $(r=.24)$. This tendency may indicate that high procrastinators reduce their procrastination levels more abstractly (as measured by IPS), whereas low procrastinators do it concretely (as measured by SRS).

\section{General discussion}

This study investigated changes in procrastination scores related to a lecture- and seminar-based intervention for reducing procrastination among first-year university students. The intervention consisted of four lectures as well as a seminar on procrastination and strategies to overcome it, all given over four weeks, as well as relevant reading material. Two measures of procrastination were used, the Irrational Procrastination Scale (IPS) and a subscale of the Self-Regulation Scale (SRS). In addition, time management skills (TMQ) and motivation to study were measured.

Overall, a significant reduction in procrastination as measured by the two scales occurred from pre- to post-test. As predicted, students high in procrastination before the intervention demonstrated the most positive change, with a significant reduction in procrastination scores, compared to little or no changes at lower procrastination levels. The scale focusing on specific procrastination behaviors (SRS) indicated a similar overall change compared to the more abstract and general scale (IPS). These results indicate that a brief intervention, such as the one reported in the present paper, can have a positive effect for students needing it most, whereas other students may demonstrate little or no benefit.

Results from the TMQ showed that students high in procrastination displayed an improved score in time management skills at the end of the intervention, while moderate and low procrastinating students demonstrated no change. In effect, reduction in procrastination was closely associated with changes in TMQ. Interestingly, high procrastinators seemed to demonstrate improved time management scores correlated with lower procrastination scores on the abstract and trait-oriented IPS scale, whereas low procrastinators demonstrated improved time management scores correlated with lower scores on the concrete (behavioral) SRS scale. This tendency indicates a potential benefit in using the SRS measure. Even if this scale may not be more sensitive in capturing short-term changes in procrastination compared to the IPS, 
it may be more sensitive in capturing real-life behavioral changes.

As expected, students with low motivation to study were found to procrastinate more than students with high motivation. However, even if high procrastinators reduced their procrastination scores and also increased their TMQ scores, no change in motivation was observed. This is understandable because no intervention efforts were specifically directed at enhancing motivation. It is also worrisome, however, because a potential side effect of the intervention might have been an increased motivation to study. Longitudinal studies may be required to capture any changes in motivation related to a reduction in procrastination.

Although positive, these results must be interpreted with caution. First, a simple pre/post design with no control group cannot document that the observed changes can be attributed to the intervention. Hence, further studies with better control should be carried out. Controlled studies should also measure the short-term as well as the longterm effects of interventions. Such studies should consider a more focused approach where students in need of help with their procrastination problem (e.g., with IPS scores > 3.0) are invited specifically. Students with moderate or low procrastination scores may feel little need to change, and smaller groups with specific needs would enable researchers to adapt course contents to such needs. Procrastinators differ (e.g., Rozental et al., 2015; Steel \& Klingsieck, 2016), and interventions adapted to specific needs may be expected to work better than general interventions. For example, some students may procrastinate because of fear of failure and worries related to highperformance standards (perfectionism), whereas procrastination in others may be related to general withdrawal and avoidance. Some students have minor-but still disadvantageous - problems with procrastination whereas others may suffer quite severely from their dysfunctional habit. Adapting interventions to specific needs may be advantageous in terms of efficacy and more appealing to the students.

A second issue complicating the interpretation of the present data is that post-data were collected toward the end of the term, when students are more compelled to work and hence to procrastinate less, aptly called the "deadline rush" (Dewitte \& Schouwenburg, 2002; König \& Kleinmann, 2005; Watson, Powell, \& Buro, 2006). However, how the "deadline rush" relates to changes in procrastination scores is not obvious. With time getting more valuable toward the end of the semester, students might feel that even small incidences of procrastination feel even worse than long delays earlier in the term when time was plentiful. If that is the case, students might report more procrastination as exams draw closer. The present data did not indicate such an increase; on the contrary, procrastination levels were stable for low and medium procrastinators and decreased in high procrastinators. Wäschle et al. (2014) observed an opposite trend to the "deadline rush" in that the students in control conditions reported less procrastination as exams approached. This may reflect a tendency to catch up, which is probably stronger for high procrastinators because they have more work to catch up on. Whether this propensity explains the change observed 
in high procrastinators in the present study remains to be settled in future studies with comparison groups of high procrastinators who do not receive an intervention.

The use of self-reported measures in evaluations of effectiveness can be problematic (Höcker et al., 2012), and high reliability of the procrastination measure is a prerequisite for detecting changes. The test/retest reliability of IPS over four months is relatively high, $r=.67$ (Steel, 2010). In the present study, test/retest reliability should be higher given only four weeks between tests. A strength of the present study is that two variants of self-reported procrastination were used. The IPS captures the general feeling of subjective delay (e.g., "I delay tasks beyond what is reasonable"), whereas SRS asks more concretely how much the respondent has delayed in the last week (e.g., "How often did you watch TV instead of studying?"). A high correlation between these two measures is reassuring, and a further benefit from using them is that they may indicate different forms of change. In the present study, the overall close correlation between TMQ changes and changes in procrastination translated into somewhat different changes in high vs. low procrastinators: High procrastinators seemed to demonstrate a cognitive or subjective change as expressed in IPS scores, whereas low procrastinators demonstrated a corresponding behavioral change as expressed in SRS scores. This may indicate that the real-life effects of interventions are better measured by instruments such as SRS and further that the outcome of intervention studies should include behavioral measures to supplement self-reported measures (Tuckman \& Kennedy, 2011).

\section{Implications and recommendations for future research}

Although the results of this study are promising, future research should further examine the causal role of intervention efforts against procrastination in academic settings. For such examination, designs with a randomized allocation of participants to conditions is preferable. Given that motivation to study is a good predictor of procrastination but-as seen in this study-is itself quite stable over time, a measure focusing on motivation to change would probably be useful, both in the recruitment process and in the prediction of potential beneficial outcome (Onwuegbuzie, 2004). Increased focus on recruitment would also allow for interventions adapted to specific needs, with potential benefits in both outcome measures and motivation to attend. Finally, although interventions against procrastination may be helpful, a preferred strategy would be to prevent dysfunctional procrastination habits to develop in the first place. This problem can be approached in two rather different ways. One is to train students not to procrastinate in preventive interventions. The other is to focus on the structural and cultural properties of student academic life, which undoubtedly represent a continuous invitation to many forms of unnecessary and irrational delay, thereby causing almost all students to procrastinate. Steel and Ferrari (2013) note that countries like Britain and the USA are exploring public policy changes to prevent procrastination, and Peru has launched government programs to reduce mañana (lateness). Future research should explore similar strategies for student academic life. 


\section{References}

Antony, M. M., Purdon, C. L., Huta, V., \& Swinson, R. P. (1998). Dimensions of perfectionism across the anxiety disorders. Behaviour Research and Therapy, 36(12), 1143-1154. doi:10.1016/S0005-7967(98)00083-7

Ariely, D., \& Wertenbroch, K. (2002). Procrastination, deadlines, and performance: Self-control by precommitment. Psychological Science, 13(3), 219-224. doi:10.1111/1467-9280.00441

Baumeister, R. F., \& Heatherton, T. F. (1996). Self-regulation failure: An overview. Psychological Inquiry, 7(1), 1-15. doi:10.1207/s15327965pli0701_1

Boice, R. (1989). Procrastination, busyness, and bingeing. Behaviour Research and Therapy, 27(6), 605-611. doi:10.1016/0005-7967(89)90144-7

Botnmark, D., Kvalnes, S., \& Svartdal, F. (2014). Prokrastinering-hvorfor studenter utsetter ting og hvordan utsettelse påvirker velvære og helse [Why students postpone things, and how this affects health and well-being]. Tidsskrift for Norsk Psykologforening, 51, 616-623.

Britton, B. K., \& Tesser, A. (1991). Effects of time-management practices on college grades. Journal of Educational Psychology, 83(3), 405. doi:10.1037/00220663.83.3.405

Dewitte, S., \& Schouwenburg, H. C. (2002). Procrastination, temptations, and incentives: The struggle between the present and the future in procrastinators and the punctual. European Journal of Personality, 16(6), 469-489. doi:10.1002/per.461

Fee, R. L., \& Tangney, J. P. (2000). Procrastination: A means of avoiding shame or guilt?. Journal of Social Behavior and Personality, 15(5), 167.

Ferrari, J. R., Johnson, J. L., \& McCown, W. G. (1995). Procrastination Research (pp. 21-46). Springer US. doi:10.1007/978-1-4899-0227-6_2

Flett, G. L., Blankstein, K. R., \& Martin, T. R. (1995). Procrastination, negative selfevaluation, and stress in depression and anxiety. In Procrastination and Task Avoidance (pp. 137-167). Springer US. doi:10.1007/978-1-4899-0227-6_7

Gallagher, R. P., Golin, A., \& Kelleher, K. (1992). The personal, career, and learning skills needs of college students. Journal of College Student Development, 33(4), 301309.

Gustavson, D. E., Miyake, A., Hewitt, J. K., \& Friedman, N. P. (2014). Genetic relations among procrastination, impulsivity, and goal-management ability implications for the evolutionary origin of procrastination. Psychological Science, 25(6), 1178-1188. 
Häfner, A., Oberst, V., \& Stock, A. (2014). Avoiding procrastination through time management: an experimental intervention study. Educational Studies, 40(3), 352360. doi:10.1080/03055698.2014.899487

Haug, I. M. (2016). Measuring Procrastination: Validation of a semi-behavioral measure. Master thesis.

Hellsten, L. A. M., \& Todd Rogers, W. (2009). Development and preliminary validation of the Time Management for Exercise Scale. Measurement in Physical Education and Exercise Science, 13(1), 13-33. doi:10.1080/10913670802609128

Höcker, A., Engberding, M., Haferkamp, R., \& Rist, F. (2012). Effectiveness of working time restriction in the treatment of procrastination. Verhaltenstherapie, 22, 9-16. doi:10.1159/000334970

Job, V., Walton, G. M., Bernecker, K., \& Dweck, C. S. (2015). Implicit theories about willpower predict self-regulation and grades in everyday life. Journal of Personality and Social Psychology, 108(4), 637. doi:10.1037/pspp0000014

Karas, D., \& Spada, M. M. (2009). Brief cognitive-behavioural coaching for procrastination: A case series. Coaching: An International Journal of Theory, Research and Practice, 2(1), 44-53. doi:10.1080/17521880802379700

Lamwers, L. L., \& Jazwinski, C. H. (1989). A comparison of three strategies to reduce student procrastination in PSI. Teaching of Psychology, 16(1), 8-12.

doi:10.1207/s15328023top1601_2

Lee, E. (2005). The relationship of motivation and flow experience to academic procrastination in university students. The Journal of Genetic Psychology, 166(1), 515. doi:10.3200/GNTP.166.1.5-15

Neenan, M. (2008). Tackling procrastination: A REBT perspective for coaches. Journal of Rational-Emotive Behavioural Therapy, 26, 53-62. doi:10.1007/s10942-007-0074-1

Ozer, B. U., Demir, A., \& Ferrari, J. R. (2013). Reducing academic procrastination through a group treatment program: A pilot study. Journal of Rational-Emotive \& Cognitive-Behavior Therapy, 31(3), 127-135. doi:10.1007/s10942-013-0165-0

Onwuegbuzie, A. J. (2004). Academic procrastination and statistics anxiety. Assessment \& Evaluation in Higher Education, 29(1), 3-19. doi:10.1080/0260293042000160384

Pychyl, T. A., Lee, J. M., Thibodeau, R., \& Blunt, A. (2000). Five days of emotion: An experience sampling study of undergraduate student procrastination. Journal of Social Behavior and Personality, 15(5), 239. 
Pychyl, T. A., \& Flett, G. L. (2012). Procrastination and self-regulatory failure: An introduction to the special issue. Journal of Rational-Emotive \& Cognitive-Behavior Therapy, 30(4), 203-212. doi:10.1007/s10942-012-0149-5

Rabin, L. A., Fogel, J., \& Nutter-Upham, K. E. (2011). Academic procrastination in college students: The role of self-reported executive function. Journal of Clinical and Experimental Neuropsychology, 33(3), 344-357. doi:10.1080/13803395.2010.518597

Roberts, M. S., Fulton, M., \& Semb, G. (1988). Self-pacing in a personalized psychology course: Letting students set the deadlines. Teaching of Psychology, 15(2), 89-92. doi:10.1207/s15328023top1502_5

Rozental, A., Forsell, E., Svensson, A., Andersson, G., \& Carlbring, P. (2015). InternetBased Cognitive-Behavior Therapy for Procrastination: A Randomized Controlled Trial. doi:10.1037/ccp0000023

Scent, C. L., \& Boes, S. R. (2014). Acceptance and Commitment Training: A brief intervention to reduce procrastination among college students. Journal of College Student Psychotherapy, 28(2), 144-156. doi:10.1080/87568225.2014.883887

Schmitz, B., \& Wiese, B. S. (2006). New perspectives for the evaluation of training sessions in self-regulated learning: Time-series analyses of diary data. Contemporary Educational Psychology, 31(1), 64-96. doi:10.1016/j.cedpsych.2005.02.002

Schraw, G., Wadkins, T., \& Olafson, L. (2007). Doing the things we do: A grounded theory of academic procrastination. Journal of Educational Psychology, 99(1), 12. doi:10.1037/0022-0663.99.1.12

Sirois, F. M. (2014). Procrastination and stress: Exploring the role of self-compassion. Self and Identity, 13(2), 128-145. doi:10.1080/15298868.2013.763404

Steel, P. (2007). The nature of procrastination: a meta-analytic and theoretical review of quintessential self-regulatory failure. Psychological Bulletin, 133(1), 65.

doi:10.1037/0033-2909.133.1.65

Steel, P. (2010). Arousal, avoidant, and decisional procrastinators: Do they exist? Personality and Individual Differences, 48(8), 926-934. doi:10.1016/j.paid.2010.02.025

Steel, P., \& Ferrari, J. (2013), Sex, education, and procrastination: An epidemiological study of procrastinators' characteristics from a global sample. European Journal of Personality, 27, 51-58. doi:10.1002/per.1851

Steel, P., \& Klingsieck, K. B. (2016). Academic procrastination: Psychological antecedents revisited. Australian Psychologist, 51(1), 36-46. doi:10.1111/ap.12173

Stöber, J., \& Joormann, J. (2001). Worry, procrastination, and perfectionism: Differentiating amount of worry, pathological worry, anxiety, and depression. Cognitive Therapy and Research, 25(1), 49-60. doi:10.1023/A:1026474715384 
Svartdal, F. (2015). Measuring procrastination: Psychometric properties of the Norwegian versions of the Irrational Procrastination Scale (IPS) and the Pure Procrastination Scale (PPS). Scandinavian Journal of Educational Research, 1-13. doi:10.1080/00313831.2015.1066439

Tice, D. M., \& Bratslavsky, E. (2000). Giving in to feel good: The place of emotion regulation in the context of general self-control. Psychological Inquiry, 11(3), 149-159. doi:10.1207/S15327965PLI1103_03

Tuckman, B. W. (1998). Using tests as an incentive to motivate procrastinators to study. The Journal of Experimental Education, 66(2), 141-147.

doi:10.1080/00220979809601400

Tuckman, B. W. \& Kennedy, G. J. (2011). Teaching learning strategies to increase success of first-term college students. Journal of Experimental Education, 79, 478504. doi:10.1080/00220973.2010.512318

Wäschle, K., Lachner, A., Stucke, B., Rey, S., Frömmel, C., \& Nückles, M. (2014). Effects of visual feedback on medical students' procrastination within web-based planning and reflection protocols. Computers in Human Behavior, 41, 120-136. doi:10.1016/j.chb.2014.09.022

Wesp, R. (1986). Reducing procrastination through required course involvement. Teaching of Psychology, 13(3), 128-130. doi:10.1207/s15328023top1303_6

Ziesat Jr., H. A., Rosenthal, T. L., \& White, G. M. (1978). Behavioral self-control in treating procrastination of studying. Psychological Reports, 42(1), 59-69. doi:10.2466/pr0.1978.42.1.59

\section{Citation}

Nordby, K., Wang, C. E. A., Dahl, T. I., \& Svartdal, F. (2016). Intervention to reduce procrastination in first-year students: Preliminary results from a Norwegian study. Scandinavian Psychologist, 3, e10. https://doi.org/10.15714/scandpsychol.3.e10

Abstract

\section{Intervention to reduce procrastination in first-year students: Preliminary results from a Norwegian study}

This paper reports preliminary results from a brief intervention designed to reduce academic procrastination. Students enrolled in an introductory psychology course received lectures and seminar sessions about procrastination and its causes and consequences. Students who were enrolled in an introductory psychology course 
received lectures and seminar sessions about procrastination and its causes and consequences, as well as information about techniques that are helpful in reducing and preventing the problem. Changes in procrastination and techniques helpful in reducing procrastination were monitored in pre and post probes. Results indicated that students reduced their procrastination, with the most positive changes observed in students who were high in procrastination tendencies at the outset of the intervention.

Keywords: academic procrastination, intervention, students.

Author affiliations: Kent Nordby, Catharina Elisabeth Arfwedson Wang, Tove Irene Dahl \& Frode Svartdal - UiT the Arctic University of Norway, Troms $\varnothing$, Norway.

Contact information: Frode Svartdal, Department of Psychology, UiT the Arctic University of Norway, N-9037 Tromsø, Norway. Email: frode.svartdal@uit.no.

Received: April 15, 2016. Accepted: June 14, 2016. Published: June 25, 2016.

Language: English.

Competing interests: The authors report no conflict of interest. The authors alone are responsible for the contents and writing of this paper.

Acknowledgement: We thank Magnus Ingebrigtsen for valuable contributions to this project.

This is a peer-reviewed paper. 